\title{
A critical review on genetics and implications of type I diabetes
}

\begin{abstract}
Type 1 diabetes is an autoimmune disorder in which the beta cells present in the pancreas which produce insulin, a hormone which mediates the levels of glucose in the blood gets damaged. After being damaged they don't produce insulin and the patient enters in the state of hyperglycemia that is elevated levels of the Blood sugars which have long complications such as ketoacidosis. The insulin is given exogenous that is through injection subcutaneously into the layer of fat just below the skin. The major susceptibility to Type 1 diabetes is researched to be HLA complex, several other genes and environmental factors. HLA complex also known MHC (major histocompatibility complex is divided into three types: class I that are encoded by HLA-A, HLA-B, and HLA-C and class II molecules are encoded by DP, DQ and DR region which is a major susceptibility factor in causing diabetes. Class III HLA complex encodes complements components and TNF factors. The long term effects of Type 1 diabetes are slow wound healing and several other associated diseases. In this review, we summarized the genetic mechanism of Type 1 diabetes and its associated complications.
\end{abstract}

Volume 7 Issue I - 2019

\section{Nida Zaman Khan, Pallabi Banerjee, Imteyaz Qamar}

School of Biotechnology, Gautam Buddha University, India

Correspondence: Imteyaz Qamar, School of Biotechnology, Gautam Buddha University, Greater Noida, Gautam Budh Nagar, UP-20I3 I 2, India, Email imteyazqamar@gmail.com

Received: December 01, 2018 | Published: January 07, 2019

\section{Introduction}

Diabetes mellitus disease study and discovery dates back to 1500BCE in Egyptian manuscript, in which they discovered that person affected by diabetes have sweet urine. ${ }^{1}$ One of the early concrete key study on Diabetes is mainly attributed to Joseph Von Mering and Oskar Minkowski. Their research was carried on dogs that showed that role of damaged pancreas leading to diabetes. ${ }^{2}$ Paul Langerhans discovered the islets of Langerhans in pancreas where insulin is produced and the role of insulin was further validated by the experiments by Sir Frederick Grant Banting and Charles Herbert Best in 1921. ${ }^{3,4}$ Diabetes mellitus are broadly classified into two types Type 1 diabetes which is insulin dependent diabetes characterized by beta cell damage in the pancreas in contrast to Type 2 diabetes which are characterized by insulin resistance where B cell is not able to release sufficient insulin. The difference between Type 1 diabetes and type 2 diabetes was first made by Sir Harold Percival (Harry) Himsworth. ${ }^{5}$

Type 1 diabetes is an immunological as well as autoimmune disease, results in the damage of beta cell present in pancreas which produces insulin. This autoimmune disorder is largely studied to be genetic which may be triggered by several environmental factors. ${ }^{5}$ Damage to the beta cells is carried upon by the CD4+ and CD8+ T cells as well as macrophages (Figure 1) ${ }^{2,7}$ Complications arise due to Type 1 diabetes are cronary heart disease, diabetic nephropathy and several others heart diseases. ${ }^{6}$ Statistical analysis showed that Type 1 diabetes is most commonly developed at childhood or adolescence ages between 0-14 years of age, but the diagnosis occurs for most of the cases is during adulthood. Averagely Type 1 diabetes affects same in both the genders. In European countries males tend to develop this disease more than females but the countries which have low incidence of Type 1 diabetes tend to have cases in which female reporting this disease higher than males. Type 1 diabetes among non-Hispanic white youth are among the highest in the world. In United States of America 1 in 300 have a chance to develop Type 1 diabetes. In India, the incidence of Type 1 diabetes has increased by $3-5 \%{ }^{25,26}$ It is researched that during the development of symptoms more than $50 \%$ of the beta cells are damaged. The auto-antigens in
Type 1 Diabetes are glutamic acid decarboxylase (GAD65), tyrosine phosphatase-like molecule (IA-2), IA-2 $\beta$ and Insulin. Some patients may have antibodies against all these auto antigens but in some cases only single antibodies are present against single auto antigen. In test subjects of newly diagnosed patients, the antibodies reported were mostly against GADA65.The antibodies against GADA65 remained stable throughout while antibodies against IA-2 tends to decrease as the disease progress.

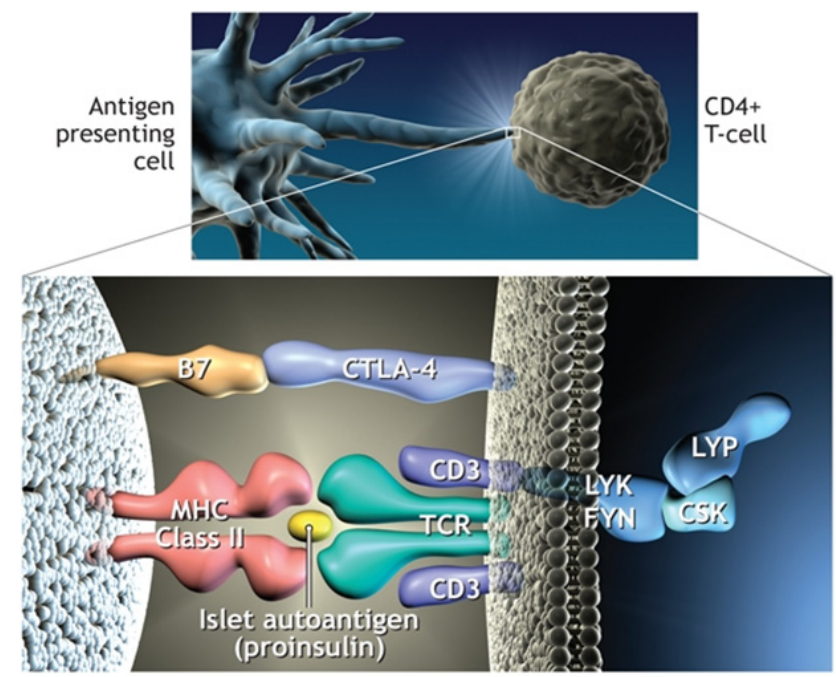

Figure I Representing T cell activation and role of genes.

\section{Symptoms}

Type 1 Diabetes is characterized by destruction of Beta cells which produce insulin in the body in response to the high glucose in the blood. The symptoms arising due to hyperglycemia (higher glucose level in the blood $>140 \mathrm{mg} / \mathrm{dl}$ ) are polyphagia (increased appetite), polydipsia (increased thirst), polyuria (increased urination), weight loss, and fatigue. 


\section{Diagnosis}

It is important to detect Type 1 Diabetes in order to start treatment. Childhood incidence of type 1 Diabetes shows the development of autoantibodies which serve as a biomarker. The detection of autoantibodies rarely occurs at six months of age, it usually occurs between the ages of 1-3 years. The autoantibodies that serve as biomarkers have been discussed above are GADA, IA-2, insulin, zinc transporter $8 .{ }^{9}$ A certain threshold is fixed that is 99 percentile in seroconversion and child must be positive for two autoantibodies in order to be diagnosed for Type 1 Diabetes. IA-2 autoantibodies are the first ones to appear and GADA are the main autoantibodies which are specific marker of type 1 Diabetes. Different antibodies have different epitopes and affinities. Sometimes low affinity antibodies are not detected by ELISA. Auto antibodies against CD4+ and CD8+ are also available in the blood along with methylated and un-methylated DNA. ${ }^{9}$ For the diagnosis of Type 1 diabetes various factors are kept in mind which is blood test at regular intervals, urine test, autoantibody test and age. The symptoms are thoroughly monitored and analyzed. First thing is the ketone body production due to ketosis is monitored which are usually excreted in urine in large quantities giving urine a brown colour. For an year urine c peptide was monitored in both adult and children, if sufficient threshold was not maintained they were classified as negative for type 1 diabetes. $^{10}$ In $2015 \mathrm{ZnT} 8 \mathrm{Ab}$ kit used to detect Type 1 Diabetes was approved by FDA which is a zinc transporter 8 auto antibody detection system. This kit was able to successfully differentiate patients of type 1 diabetes from type 2 diabetes. $^{2}$

\section{Implications}

\section{Diabetic ketoacidosis}

Diabetic Ketoacidosis is a complication which arises due to Type 1 Diabetes and in some cases patients suffering from Type 2 Diabetes is also experience it. Ketoacidosis arises due to insulin deficiency. Without insulin, glucagon hormone doesn't get inhibited resulting in the higher levels of glucagon, free fatty acids and amino acids in blood. ${ }^{11}$ Increase of cortisol, glucagon, growth hormone, and catecholamines also occurs. Due to the lack of production of insulin, body can't use glucose as energy, alternatively fat breakdown occurs which leads to production of free fatty acids and some of the fatty acids form acetyl coenzyme A that enter the kreb cycle for energy production. Conversely production of ketones such as $\beta$-hydroxybutyrate ( $\beta$-OHB), acetoacetate, and acetone also occur by the breakdown of fatty acids. Also Hyperglycemia occurs due to ketoacidosis and its complication causes osmotic diuresis. Osmotic diuresis leads to dehydration. The other complications are hyperosmolarity, electrolyte loss, and subsequent decrease in glomerular filtration. Potassium uptake in muscle cells also decreases leading in overall loss of potassium. ${ }^{12}$ Complication involving DKA are Hamman's syndrome which is a respiratory ailment. Symptoms of Hanman's syndrome include chest pain, shortness of breath, neck pain and popping sound during systole. ${ }^{13}$

\section{Impaired wound healing}

Studies on mice showed the development of LGI (low grade inflammation) by leukotriene (LT) in Diabetic patients. LG1 is involved in impaired wound healing whereas the increased levels of leukotriene are the product of enzyme 5-lipoxigenase (5LO). Enzyme 5-lipoxigenase (5LO) forms leukotriene by acting upon arachidonic acid (AA). There are two types of macrophages one which promotes anti-inflammatory process that is M2 macrophages other types of macrophages that promote inflammatory process. M2 macrophages promote wound healing by promoting angiogenesis and collagen deposition. Leukotriene was shown to control macrophages which delayed the immune response and in turn the cytokine production continued which hence delays the healing process as longer the inflammatory response continues it in turns slows the wound healing process. Studies were carried on diabetic mice where two types of mice were taken which produced the enzyme 5-lipoxigenase (5LO) and the other mice which didn't express the enzyme 5-lipoxigenase (5LO). The mice which were wild type diabetic produced higher number of cytokine TNF- $\alpha$, IL-6, IL-10, IL-12 and IFN $\gamma$ pro-inflammatory monocytes increased expression of classically activated macrophages, such as iNOS (encoded by Nos2 gene), Stat1 and I112). The mice lacking (5L0) expressed higher ym1, arg1 and arginase production which is correlated with wound healing. A higher level of leukotriene was observed in wild type diabetic mouse. Physical analysis of wound healing was also carried out in the above two types of mice even though there was delay observed in wound healing in mice lacking $5 \mathrm{LO}$, all the wounds were closed within 18 days unlike the wild type diabetic where the wounds were 15 percent of the original size . The physical analysis was supported by area under the curve. ${ }^{8}$

\section{Genetics}

\section{HLA}

The precise location of HLA is 6p21.3 on chromosome 6. HLA is researched to be largely associated with Type1 Diabetes. HLA contributes $40-50 \%$ to predisposition of type1 Diabetes. The locus identified is class II genes HLA-DR, DQ, DP which are majorly associated with causing type1 Diabetes. Many other loci are associated with Diabetes have also been discovered. Individuals having haplotype DRB1-DQA1-DQB1 are significantly at a higher risk of developing type 1 diabetes. ${ }^{20}$ The genes present in HLA code for Class I and Class II molecules. IDDM1 is another name by which HLA class II region is known .Class I is coded by A, B and C and Class II is coded by DP, DQ and DR regions. HLA coding Class II molecules are expressed by antigen presenting cells and express antigen to CD4+ T cells. In experiments done on Non-Obese mouse only one peptide was discovered $\mathrm{I}-\mathrm{Ag} 7$ which acts site for the action of CD4+ T cells. This was proved by deletion or replacement of MHC alleles coding for I-Ag7 in NOD mouse. ${ }^{14}$

$\mathrm{I}-\mathrm{Ag} 7$ has the same alpha chain structure as present in non IIDM linked alleles but presence of unique beta chain which have unique 17 residues confers the diabetogenic character specially two residues Hisb56 and Serb57 which are polymorphic and when replaced by Prob56 or Aspb5 reduces diabeteic susceptibility. ${ }^{19}$ The nonaspartic acid residue at position 57 of their $\beta$ chain in MHC alleles removes the salt bridge between Arg $\alpha 76$ and $\beta 57$ which eliminates the negative charge, altering the P9 pocket of the peptide binding groove which results in a positive charge on MHC and This is detected by TCR. The positive surface on the P9 in I-Ag7 was accessible and detected by negative residue on the TCR. ${ }^{15}$

\section{INS GENE}

Insulin gene (INS) is located in the IDDM2 Locus. The exact locus of this gene is $11 \mathrm{p} 15.5$.Variable number of tandem repeats present upstream of INS consist of polymorphism which are responsible for type 1 Diabetes. There are class 1, 2, and 3 alleles present .Class 1 allele is responsible for causing Type 1 Diabetes. The class 1 alleles express high levels of insulin in pancreas but express low levels in thymus and class 3 alleles perform vice versa. Low levels of insulin 
promote $\mathrm{T}$ cells $\mathrm{CD}+4$ activation. High levels of insulin promote protection from type 1 Diabetes. ${ }^{16}$

\section{CTLA4}

This gene is located on chromosome 2. Specific locus of CTLA 4 gene is long arm of 2q33.CTLA 4 binds B7 molecule present in the Antigen Presenting Cells (APC) which stimulates the $\mathrm{T}$ cell activation. CTLA 4 plays an important role in regulation of $\mathrm{T}$ cell activation by providing negative signal to inhibit $\mathrm{T}$ cell activation. Mutations in CTLA4 region cause an increase in the stimulation in $\mathrm{T}$ cell activation. A49G polymorphism in CTLA4 is proved to play a direct role in development of Type 1 Diabetes. After meta-analysis on a mixed population was performed, at exon 1 of CTLA4 transition from nucleotide $\mathrm{A}$ to $\mathrm{G}$, causes a change in amino acid threonine to alanine. The other polymorphism found are $\mathrm{C}$ to $\mathrm{T}$ substitution at 318 region of the promoter and A to $\mathrm{G}$ Transition at exon 1 position $49 .{ }^{17}$

\section{PTPN22}

PTPN22 encodes lymphoid tyrosine phosphatase (LYP), located on chromosome number 1. Lymphoid tyrosine phosphatase (LYP) dephosphorylases kinases Lck, Fyn, ITAMS of the Src family present in the TCR/CD3 complex. The amino acid at position 620 changes from $\operatorname{Arg}(\mathrm{R})$ to $\operatorname{Trp}(\mathrm{W})$ which is a missense mutation .The change occurring causes chk gene not to bind and hence causes $\mathrm{T}$ cell activation. ${ }^{18-20}$

\section{Environmental factors}

Viruses are researched to be a potential cause of type 1 Diabetes. Enteroviruses have strongest correlation as in some studies immune response against VIP produces PKR and myeloid cell factor which in turn leads to apoptosis. During pregnancy infection with enteroviruses may also lead to damage to Beta cells due to autoimmunity in both mother and child is also postulated. Dietary habit is also studied such as breastfeeding. A study by ABIS in Sweden shows infants who had breast-milk even when cereals were introduced were somehow resistance to type 1 diabetes. Studies show that cow milk ingestion during infancy increased a risk of autoimmunity in Beta cells. Casein hydrolysate could be substituted against breast milk but cow milk did make the infant susceptible to type 1 Diabetes. This study was done on infants who postulated that components in cow's milk that were fatty acids such as myristic, penta-decanoic, and mono-unsaturated palmitoleic acid isomers 16:1 omega-7 and 16:1 omega-9 and conjugated linoleic acid were the cause of Type 1 Diabetes but this theory has to be proven yet. Vitamin D is an essential vitamin for the human body and regulates many aspects of immune system of the body. The response to $\mathrm{T}$ cell on down-regulation of the T-helper-1 immune response is mediated by vitamin D. Many studies occurring at different demographics were conducted to establish relationship between vitamin D and Type1 Diabetes. Vitamin D intake during pregnancy showed no relationship of type 1 diabetes and vitamin D but a study in Norway showed taking of vitamin D in late stages of pregnancy did make the infant less susceptible to Type 1 Diabetes. The other environmental factors proposed and researched to be linked with type 1 diabetes are microbial population in intestine also known as intestinal microbiota Some reports show lower level of intestinal microbiota were present before progressing to type 1 Diabetes but more research has to be done. The BCG vaccine is thought to protect from autoimmunity but not many conclusive results have come up. ${ }^{21}$

\section{Conclusion}

In this review we tried to summarize the genetic and environmental factors that are major factors in predisposition of Type I Diabetes. Further research can be done on genetic and cell signalling mechanism of I-Ag7 peptide in mouse. Similar peptides in humans have to be discovered which act as a site of CD4+ cells. The mutations causing type 1 diabetes can be studied and their expression in real time has to be monitored. Another mechanism of insulin delivery to Type 1 Diabetic patients has to be administrated which may be less invasive and painful than injection.

\section{Acknowledgements}

None.

\section{Conflict of interest}

Author declares that there is no conflict of interest.

\section{References}

1. Leonid P. Principles of diabetes mellitus. ( $2^{\text {nd }}$ ed.). New York: Springer. 2000; p. 3. ISBN: 978-0-387-09840-1.

2. Von Mehring J, Minkowski O. Diabetes mellitus nach pankreasexstirpation. Archiv für experimentelle Pathologie und Pharmakologie. 1890;26(5-6):371-387.

3. Bryan J. Just the Facts Diabetes. Chicago, Illinois: Heinemann Library a division of Reed Elsevier Inc. 20004; p. 7.

4. Linda B, Courtney H. Commentary: More than 'tentative opinions': Harry Himsworth and defining diabetes. International Journal of Epidemiology. 2013;42(6):1599-1600.

5. Paschou SA, Papadopoulou-Marketou N1, Chrousos GP, et al. On type 1 diabetes mellitus pathogenesis Endocr Connect. 2018;7(1):R38-R46.

6. Bjornstad P, Snell-Bergeon JK, Nadeau KJ, et al. Insulin sensitivity and complications in type 1 diabetes: New insights. World $J$ Diabetes. 2015;6(1): 8-16.

7. Notkins AL, Lernmark A. Autoimmune type 1 diabetes: resolved and unresolved issues. J Clin Invest. 2001;108(9):1247-1252.

8. Ramalho T, Filgueiras L, Silva-Jr IA, et al. Impaired wound healing in type 1 diabetes is dependent on 5-lipoxygenase products. Sci Rep. 2018;8(1):14164

9. Bonifacio E. Predicting Type 1 Diabetes Using Biomarkers. Diabetes Care. 2015;38(6):989-996.

10. National Collaborating Centre for Women's and Children's Health (UK). Diabetes (Type 1 and Type 2) in Children and Young People: Diagnosis and Management. London: National Institute for Health and Care Excellence.

11. Raghupathy P. Diabetic ketoacidosis in children and adolescents. Indian $J$ Endocr Metab. 2015;19(Suppl S1):55-57.

12. Gosmanov AR, Gosmanova EO, Kitabchi AE. Hyperglycemic Crises: Diabetic Ketoacidosis (DKA), and Hyperglycemic Hyperosmolar State (HHS). In: De Groot LJ, Chrousos G, Dungan K, et al., editors. Endotext. South Dartmouth (MA): MDText.com, Inc.; 2000.

13. Pain AR, Pomroy J, Benjamin A, et al. Hamman's syndrome in diabetic ketoacidosis. Endocrinol Diabetes Metab Case Rep. 2017;2017:170135 .

14. Zhao Y, Scott NA, Quah HS, et al. Mouse pancreatic beta cells express MHC class II and stimulate CD4+ T cells to proliferate. Eur J Immunol. 2015;45(9):2494-2503.

15. Yoshida K, Corper AL, Herro R, et al. The diabetogenic mouse MHC class II molecule I-Ag7 is endowed with a switch that modulates TCR affinity. J Clin Invest. 2010;120(5):1578-1590. 
16. Pugliese A. The insulin gene in type 1 diabetes. IUBMB Life. 2005;57(7):463-468.

17. Kavvoura FK, Ioannidis JP. CTLA-4 Gene Polymorphisms and Susceptibility to Type 1 Diabetes Mellitus: A HuGE Review and MetaAnalysis. Am J Epidemiol. 2005;162(1):3-16.

18. Saccucci P, Del Duca E, Rapini N, et al. Association between PTPN22 C1858T and type 1 diabetes: a replication in continental Italy Association between PTPN22 C1858T and type 1 diabetes: a replication in continental Italy. Tissue Antigens. 2008;71(3):234-237.

19. Corper AL, Stratmann T, A postolopoulos V, et al. A Structural Framework for Deciphering the Link Between I-Ag7 and Autoimmune Diabetes. Science. 2000;288(5465):505-511.

20. Noble JA, Valdes AM. Genetics of the HLA Region in the Prediction of Type 1 Diabetes. Curr Diab Rep. 2011;11(6):533-542.

21. Rewers M, Ludvigsson J. Environmental risk factors for type 1 diabetes. Lancet. 2016;387(10035):2340-2348.

22. Mahajan R. Blood test to help diagnose type 1 diabetes approved by Food and Drug Administration. Int J Appl Basic Med Res. 2015;5(1):1.
23. Corsino L, Dhatariya K, Umpierrez G. Management of Diabetes and Hyperglycemia in Hospitalized Patients. In: De Groot LJ, Chrousos G, Dungan K, et al., editors. Endotext [Internet]. South Dartmouth (MA): MDText.com, Inc.; 2000.

24. Simmons KM, Michels AW. Type 1 diabetes: A predictable disease. World J Diabetes. 2015;6(3):380-390.

25. Ashok Kumar Das. Type 1 diabetes in India: Overall insights Indian. $J$ Endocrinol Metab. 2015;19(Suppl 1):S31-S33.

26. David Maahs DM, West NA, Lawrence JM, et al. Epidemiology of Type 1 Diabetes. Endocrinol Metab Clin North Am. 2010;39(3):481-497.

27. Gillespie KM. Type 1 diabetes: pathogenesis and prevention. CMAJ. 2006;175(2):165-170.

28. Kahn SE, Cooper ME, Del Prato S. Pathophysiology and treatment of type 2 diabetes: perspectives on the past, present, and future. Lancet. 2014;383(9922):1068-1083.

29. Ndisang JF, Vannacci A, Rastogi S. Insulin Resistance, Type 1 and Type 2 Diabetes, and Related Complications 2017. J Diabetes Res. 2017;2017:1478294. 